\title{
UAV-BASED ACQUISITION OF 3D POINT CLOUD - A COMPARISON OF A LOW-COST LASER SCANNER AND SFM-TOOLS
}

\author{
D. Mader*, R. Blaskow, P. Westfeld, H.-G. Maas \\ Technische Universität Dresden, DE-01062 Dresden, Germany \\ Institute of Photogrammetry and Remote Sensing \\ (david.mader, robert.blaskow, patrick.westfeld, hans-gerd.maas)@tu-dresden.de \\ http://www.tu-dresden.de/ipf/photo
}

KEY WORDS: Unmanned aerial vehicle, 3D point cloud, LIDAR, structure from motion, laser scanning range finder, RGB camera

\begin{abstract}
:
The Project ADFEX (Adaptive Federative 3D Exploration of Multi Robot System) pursues the goal to develop a time- and cost-efficient system for exploration and monitoring task of unknown areas or buildings. A fleet of unmanned aerial vehicles equipped with appropriate sensors (laser scanner, RGB camera, near infrared camera, thermal camera) were designed and built. A typical operational scenario may include the exploration of the object or area of investigation by an UAV equipped with a laser scanning range finder to generate a rough point cloud in real time to provide an overview of the object on a ground station as well as an obstacle map. The data about the object enables the path planning for the robot fleet. Subsequently, the object will be captured by a RGB camera mounted on the second flying robot for the generation of a dense and accurate $3 \mathrm{D}$ point cloud by using of structure from motion techniques. In addition, the detailed image data serves as basis for a visual damage detection on the investigated building.

This paper focuses on our experience with use of a low-cost light-weight Hokuyo laser scanner onboard an UAV. The hardware components for laser scanner based 3D point cloud acquisition are discussed, problems are demonstrated and analyzed, and a quantitative analysis of the accuracy potential is shown as well as in comparison with structure from motion-tools presented.
\end{abstract}

\section{INTRODUCTION}

Unmanned Aerial Vehicles (UAVs) are small and versatile aircrafts at a reasonable price. Their modular structure allows simple and time-saving adjustments for certain requirements and simplifies the maintenance. Due to these characteristics, UAVs find large interest as a tool for a wide spectrum of civil applications such as exploration, monitoring, inspection or rescue tasks as well as large-scale aerial photogrammetry. The interdisciplinary Project ADFEX (Adaptive Federative 3D Exploration of Multi Robot Systems) shall carry out the ambitious goal of data acquisition for exploration and monitoring tasks by an autonomous operating group of flying robots. Three robots have been developed and constructed using standard sensors for navigation and for obstacle detection (GPS, IMU, ultrasonic sensors). In addition, the UAVs are equipped with special sensors for acquisition of different tasks, which can be adapted to different applications. A laser scanning range finder (LSRF) enables a real-time generation of $3 \mathrm{D}$ point clouds with reduced accuracy to provide an overview of the object and to facilitate obstacle mapping and path planning. Images captured with RGB-, near-infrared and thermal-infrared cameras form a basis for the reconstruction of more accurate $3 \mathrm{D}$ point clouds by using structure from motion (SfM) processing methods. A cost and time efficient exploration and monitoring should be achieved by a simultaneous task-sharing flight of the three robots with a continuous two-way data stream between the robots and the ground station. More information can be found on the website http://www. adfex.eu/. Other studies also attend on UAVs equipped with light-weight imaging sensors. In (Ahmed et al., 2008) UAVs were utilized as aircrafts, which were flying at a very low altitude to get high resolution images of the ground. Based on these images, victims in disaster areas can be detected and rescued. Israel (2011) described a thermal detection system for saving lives of fawns in meadows on basis of images captured by a thermal camera mounted on an UAV. An

${ }^{*}$ Corresponding author efficient usage for monitoring of soil erosion is shown in (Eltner et al., 2013), where an UAV equipped with a camera was used for data acquisition. The authors in (Roca et al., 2014) produced 3D point clouds for building assessment with a combination of light-weight laser scanning range finder and an unmanned aircraft. A processing chain for operator guidance through an UAV based building reconstruction by the help of SfM techniques is described in (Daftry et al., 2015).

This paper will focus on our experience in generating 3D point clouds from low-cost laser scanner data in comparison to SfM techniques on basis of RGB images, both mounted on UAVs. The first part presents a brief overview about the specification of the sensors mounted on the UAV and the flying platform itself. The second part deals with the acquisition and processing of 3D point clouds as well as an analysis of the results. Finally, this constribution will finish with a conclusion, an outlook and future work.

\section{HARDWARE}

\subsection{Unmanned Aerial Vehicle}

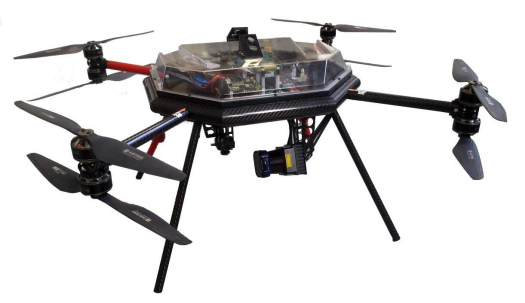

Figure 1: UAV Cadmic Goliath Coax 8 frame.

As part of the project ADFEX, three flying robots have been designed and built. The basic construction of each robot consists of a Cadmic Goliath Coax 8 frame with four cantilever arms and 


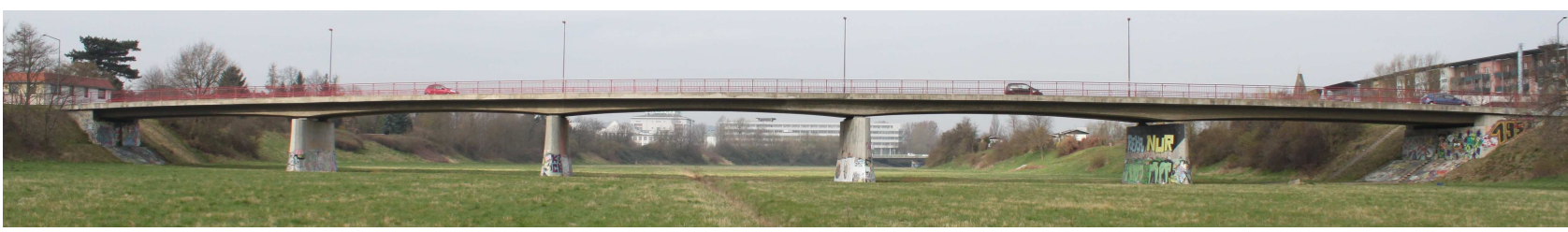

(a)

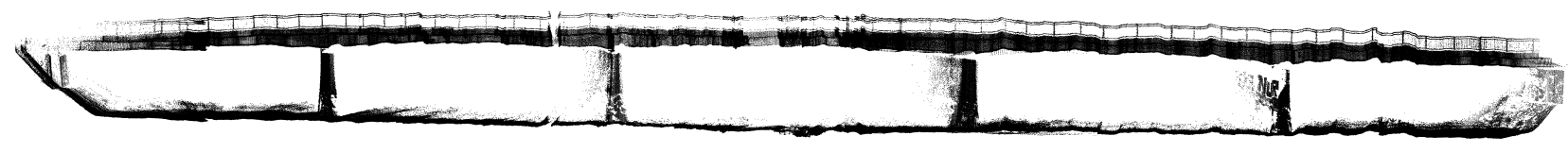

(b)

Figure 2: (a) Image of the investigated bridge. (b) Overview of the entire 3D point cloud based on data from LSRF.

eight coaxial mounted rotors (Figure 1). For the determination of the robots position and orientation, the raw data of a singlefrequency GPS-receiver u-blox LEA-6T and an IMU-module 10DOF-IMU ADIS16407 are processed. The advantage of the modular structure of the robots in comparison to black box systems is the easy access to every component in case of maintenance or emergency. The payload is about $1 \mathrm{~kg}$, which is sufficient in order to carry passive or active 2D/3D sensor systems at a reasonable flight time of about $10 \mathrm{~min}$ to $15 \mathrm{~min}$ per battery charge.

\subsection{Hokuyo UTM-30LX-EW}

One of the platforms is equipped with a laser scanner UTM30LX-EW from Hokuyo (Figure 3a). It is a 2D laser scanning range finder (LSRF), capturing distances in a plane. The distance measurement unit operates according to the time-of-flight principle. Due to the low weight of only $210 \mathrm{~g}$ and the compact size $(62 \mathrm{~mm} \times 62 \mathrm{~mm} \times 87.5 \mathrm{~mm})$, the LSRF is predestinated to be used on UAVs. The field of view is $270^{\circ}$ with a resolution of $0.25^{\circ}$, which results in 1080 distance measurement per line during a $1 / 40$ seconds. The LSRF works in a range of $0.1 \mathrm{~m}$ up to $30 \mathrm{~m}$ with an accuracy of $30 \mathrm{~mm}$ to $50 \mathrm{~mm}$ (specified by manufacturer). An important fact for subsequent error analysis is the elliptical laser footprint with a beam divergence of about $1.0^{\circ} \times 0.1^{\circ}$, which is rotating during a scan (Figure $3 \mathrm{~b}$ ).

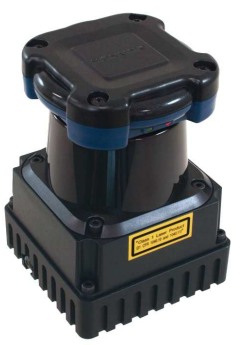

(a)

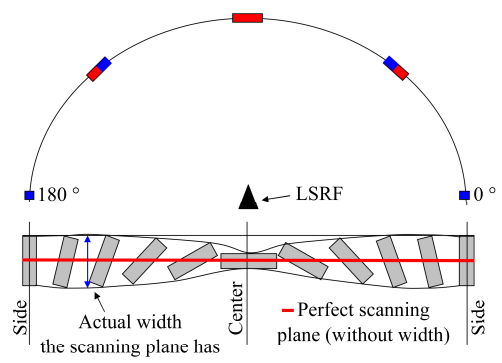

(b)
Figure 3: (a) Laser scanning range finder Hokuyo UTM-30LXEW (Hokuyo, 2014a). (b) Schematic representation of the laser beam footprint (specified by manufacturer); the figure shows the effect of a rotating elliptical laser beam profile (Hokuyo, 2014b).

\subsection{AVT Prosilica GT3300C}

For high quality image acquisition, a CCD camera $3296 \times 2472$ px Prosilica GT3300C (Figure 4) from Allied Vision Technologies is used. The pixel size is $5.5 \mu \mathrm{m}$, and the maximum frame rate is 14.7 images per second. With a weight of $314 \mathrm{~g}$ for the camera body and dimensions of $121 \mathrm{~mm} \times 59.7 \mathrm{~mm} \times 59.7 \mathrm{~mm}$, it is still suitable for use on UAVs with a sufficient payload. For the capture in this contribution, a lens with a fixed focal length of $20 \mathrm{~mm}$ was used. This sensor-lens combination (crop factor $=1.91$ ) is equivalent to a full format sensor and a focal length of $38.19 \mathrm{~mm}$.

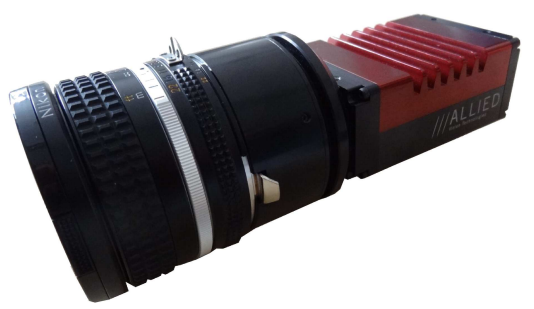

Figure 4: Prosilica GT3300C RGB camera.

\section{CALIBRATION}

To receive high-quality results, a calibration of the entire system and the sensors is indispensable. An entire system calibration comprises the lever arm as well as the boresight angles between the coordinate systems of the LSRF, the camera and the UAV's navigation sensors. The sensor calibration also includes the interior parameters such as camera lens distortions or distance corrections for the LSRF. Both were carried out simultaneously in an integrated self-calibration strategy to guarantee a correct calculation with different observation types. An adaptive fully integrated and modularly structured bundle adjustment is shown in (Mader et al., 2014), which was extended with geometric and stochastic models for the determination of lever arm and boresight angles for the particular sensor-UAV combination.

\section{3D POINT CLOUDS BASED ON LSRF DATA}

\subsection{Acquisition and data processing}

As research object a bridge (Figure 2a) with dimensions of $12 \mathrm{~m}$ in width, $135 \mathrm{~m}$ in length and $8 \mathrm{~m}$ in height, was selected. Scans alongside the bridge with low speed of the UAV guaranteed a sufficient number of scan lines for a good representation of the bridge in LSRF data. In total 20,000 scan lines were recorded in a flight-time of approximately $10 \mathrm{~min}$ with an average altitude of $5 \mathrm{~m}$ and an average UAV-object distance of $12 \mathrm{~m}$. Additional flights underneath the bridge were also flown for test purposes, 


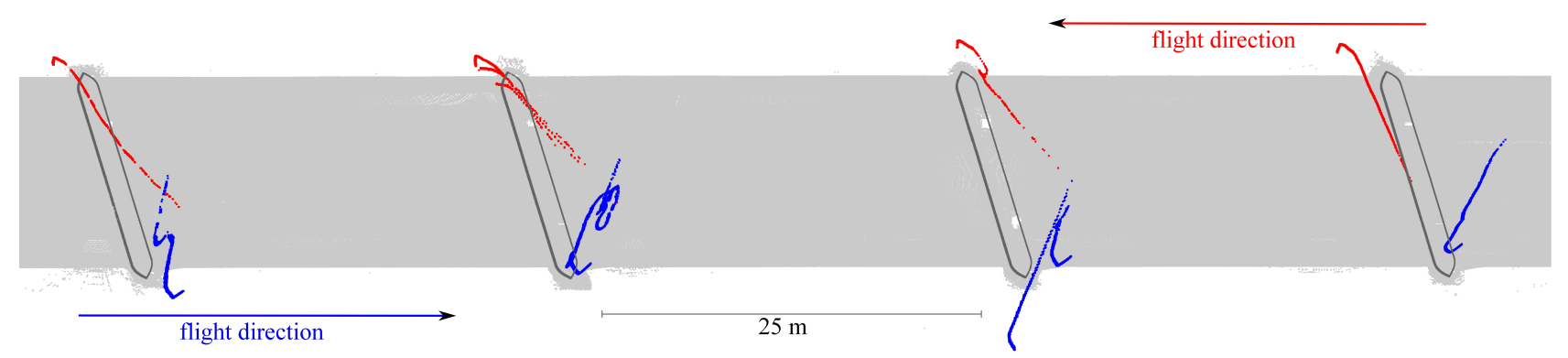

(a)

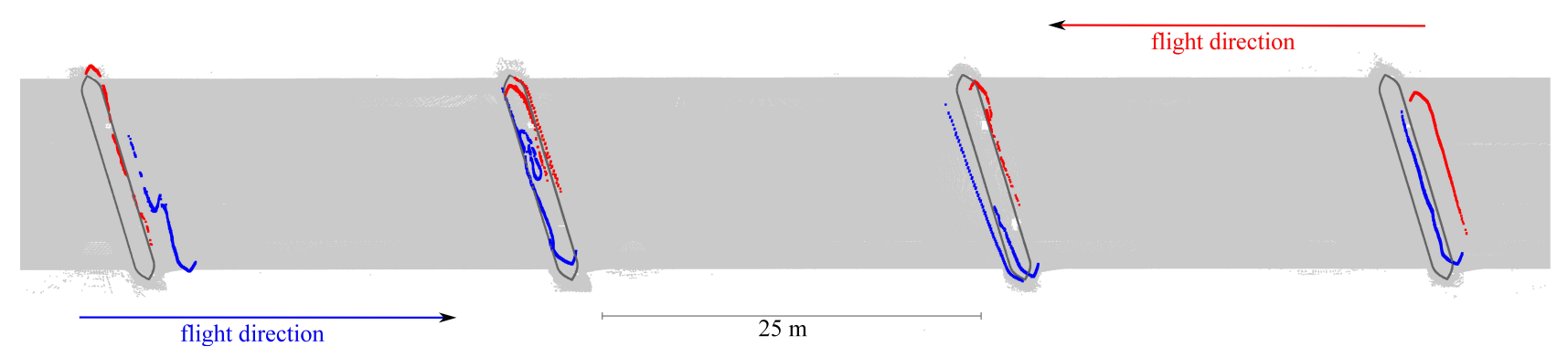

(b)

Figure 5: Horizontal profile of pillars from TLS (gray)- and LSRF (blue, red) point cloud with: (a) incorrect yaw angle measurements. (b) corrected yaw angles by approximate correction terms. The pillars are $14.1 \mathrm{~m}$ in length and $1.4 \mathrm{~m}$ in width.

but as expected the LSRF measurement could not be used due to GPS signal loss. As stated above, the LSRF provides 40 scan lines per second, each with 1080 distance and intensity measurements. Each scan line of the LSRF and the UAV pose information gets a timestamp for synchronizing the acquired data. Due to the fact that the LSRF and navigation sensors are not hardwaretriggered and thus not congruent, the pose of every scan line had to be linear interpolated depending on the timestamps. For 3D point cloud generation, the first step is the conversion from measured spherical coordinates (distance $D$, horizontal angle $\alpha$, vertical angle $\beta$ ) into the local laser scanner coordinate system respectively the sensor coordinate system (scs). In this particular case (the used LSRF performs 2D scans) the vertical angle component $\beta$ and thus the resulting Z-component are zero in the scs system.

$$
\mathbf{X}^{\mathrm{scs}}=\left[\begin{array}{c}
X^{\mathrm{scs}} \\
Y^{\mathrm{scs}} \\
Z^{\mathrm{scs}}
\end{array}\right]=D \cdot\left[\begin{array}{c}
\cos \alpha \\
\sin \alpha \\
\sin \beta
\end{array}\right]
$$

$$
\begin{array}{ll}
\text { where } & \\
D & : \text { measured distance from LSRF } \\
\alpha & : \text { horizontal deflection angle } \\
\beta & : \text { vertical deflection angle }(:=0)
\end{array}
$$

Subsequently, the point coordinates are transformed into the UAV coordinate system (rcs - robot coordinate system) by using the calibrated lever arm and boresight angles.

$$
\mathbf{X}^{\mathrm{rcs}}=\mathbf{X}_{\mathrm{la}}+\mathbf{R}_{\mathrm{scs}}^{\mathrm{rcs}} \cdot \mathbf{X}^{\mathrm{scs}}
$$

$$
\begin{array}{ll}
\text { where } & \\
\mathbf{X}_{\text {la }} & \text { : lever arm in roboter coordinate system (rcs) } \\
\mathbf{R}_{\text {scs }}^{\text {rcs }} & \text { : rotation matrix of boresight angles } \\
\mathbf{X}^{\text {rcs }} & \text { : object point in roboter coordinate system (rcs) }
\end{array}
$$

Finally, on basis of the navigation data the measured points are mapped into the WGS84 reference frame. For that the point coordinates are transformed into the navigation system (north-east- down coordinate system - ned), with

$$
\mathbf{X}^{\mathrm{ned}}=\mathbf{R}_{z}(\psi) \cdot \mathbf{R}_{y}(\theta) \cdot \mathbf{R}_{x}(\phi) \cdot \mathbf{X}^{\mathrm{rcs}}
$$

where

$\phi, \theta, \psi \quad$ : orientation angles based on IMU measurements

$\mathbf{X}^{\text {ned }} \quad$ : object point in navigation coordinate system (ned)

and from north-east-down in the WGS84 (wcs - world coordinate system) with:

$$
\mathbf{X}^{\mathrm{wcs}}=\mathbf{X}_{\mathrm{ned}}^{\mathrm{wcs}}+\left(\mathbf{R}_{y}\left(\text { lat }+\frac{\pi}{2}\right) \cdot \mathbf{R}_{z}(\text { lon })\right)^{T} \cdot \mathbf{X}^{\mathrm{ned}}
$$

where

lat : geographical latitude

lon : geographical longitude

$\mathbf{X}^{\text {wcs }} \quad$ : object point in WGS84 coordinate system (wcs)

$\mathbf{X}_{\text {ned }}^{\text {wcs }}:$ UAV-position in WGS84 coordinate system (wcs)

The final coordinates results from an addition with the corresponding UAV-position. A detailed description of the transformation between the robot coordinate system and the WGS84 can be found in (Bäumker and Heimes, 2001).

\subsection{Result}

The generated 3D point cloud (Figure $2 \mathrm{~b}$ ) was geo-referenced by the LSRF distance measurement unit as well as the UAV GPSposition and includes about 1 million points (bridge only). Point spacing in scan line direction was approximately $5 \mathrm{~cm}$ for an object distance of about $10 \mathrm{~m}$. Due to the quite steady and slow flying speed, the line distance reached an average of $6 \mathrm{~cm}$, which resulted in a point density of about 333 points per squares meter. Important object details such as bridge pillars, the roadway and the general shape of the bridge are well recognizable in the data. Errors in the LSRF and navigation sensor data as well as in the lever arm and boresight angles have a direct influence on the quality of the $3 \mathrm{D}$ point cloud. GPS and IMU measurement errors have the largest influence, whereas the error in the LSRF 


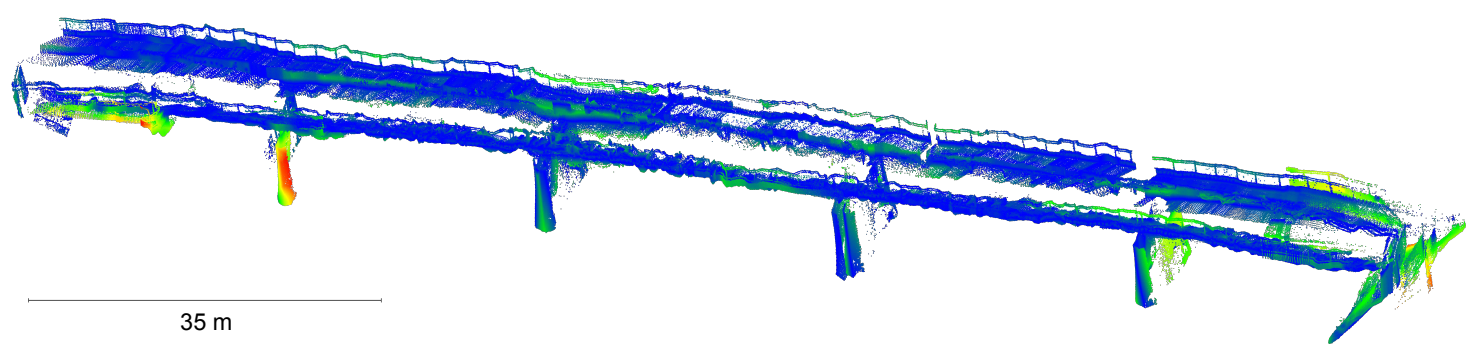

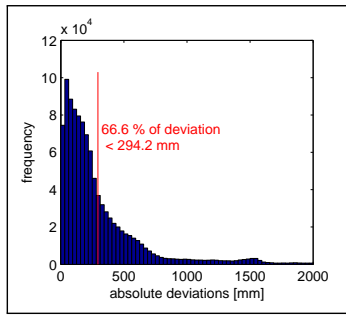

(b)

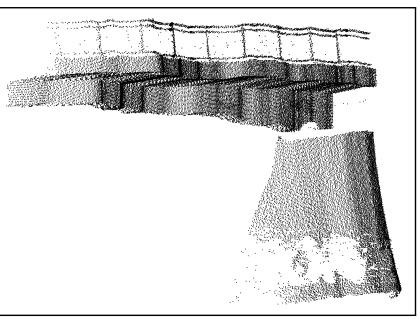

(c) (a)

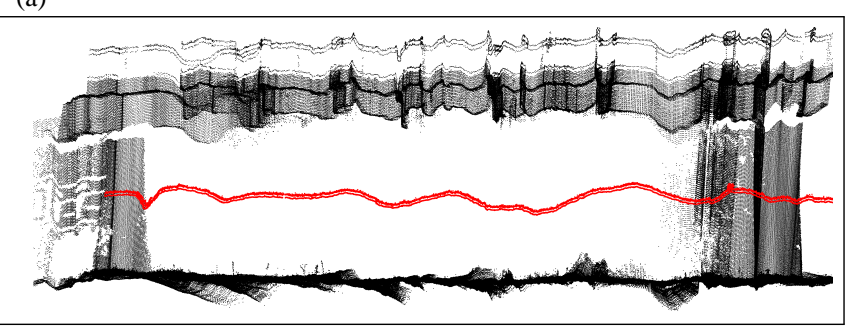

(d)

Figure 6: (a) Color-coded absolute distances between LSRF and TLS point clouds obtained by CloudCompare. (b) Histogram of absolute distances. (c) Good represented part of the bridge by LSRF points. (d) Bad represented part of the bridge by LSRF points caused by inaccurate IMU angles in lateral direction. Red points are the flight path of the UAV.

distances $(\sim 30 \mathrm{~mm}$ to $50 \mathrm{~mm})$ is negligible. The lever arm and boresight angles, which were determined by an appropriate calibration strategy, have also a low influence. The weakness is obviously the accuracy of measured GPS-heights (Figure 7a), however it can be improved by an additional barometric altitude measurement, as one can see in Figure 7b. In Figure 5a, a horizontal section through the bridge pillars is shown to illustrate the impact of inaccurate yaw angles. This effect was probably caused by the influence of electromagnetic fields from the UAV's electronic components to the magnetometer. The torsion is clearly evident in comparison to a terrestrial laser scanner (TLS) point cloud, which was captured with a high-precision Z\&F Imager 5006 laser scanner and used as reference. A rough correction term for the yaw angles was determined by an alignment of the pillars horizontal profile from LSRF point cloud to the TLS point cloud and considered in the processing chain of UAV point cloud generation (Figure 5b). The corrected point cloud still contains lateral GPS-inaccuracies. For quantifying this fact, a comparison between the LSRF and TLS point clouds was done. Therefor, the LSRF point cloud was transformed in two steps onto the TLS point cloud. First step is a coarse alignment with three better more homologous points and second step is the fine registration by an iterative closest point (ICP) algorithm. Afterwards the cloud to cloud distances were calculated. The result shows a sufficient inner geometrical accuracy for a rough point cloud (Figure 6a). The LSRF point cloud shows a deviations root mean square value (RMS) of about $31.7 \mathrm{~cm}$, with $2 / 3$ of all points having deviations less than $29.4 \mathrm{~cm}$. Figure $6 \mathrm{~b}$ show the distribution of the deviation compared to the TLS point cloud. These lateral effects might be reduced by a stronger weighting of the IMU acceleration measurements in the GPS/IMU data processing. Other GPS based approaches such as real time kinematic (RTK) or differential GPS (DGPS) are also possible shown in (Stempfhuber and Buchholz, 2011) and (Heredia et al., 2009). The expected 3D platform position accuracy is about few centimeter for both. However, RTK and DGPS navigation are not robust for signal loss caused by shadowing effects. Due to this fact, an additional improvement of the used navigation solution may be obtained from sensors like a navigation camera in a visual SLAM approach similar to (Georgiev and Allen, 2004). A further as- pect for enhancement would be an appropriate magnetometer calibration as described in (Gebre-Egziabher, 2007, Dorveaux et al., 2009, Metni et al., 2006) or improved protection against signal interfering radiation through EMI shields. Figure $6 \mathrm{~d}$ discloses a major problem caused by direct dependencies of navigation solution, in this case the IMU angles. Nevertheless, Figure 6c shows a well captured bridge part and confirms the potential of this capturing system benefiting from smaller direction changes of the UAV. Despite the above mentioned problems, the generated 3D point cloud is suitable for a good overview of the object or for obstacle avoidance.

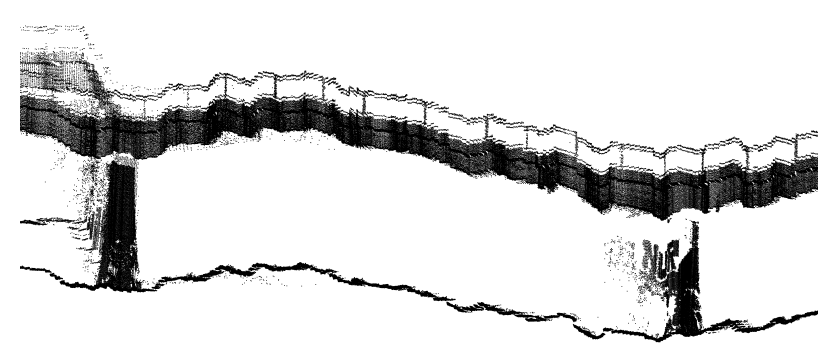

(a)

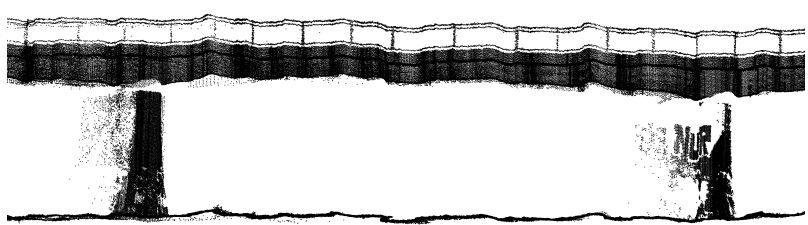

(b)

Figure 7: Effects of inaccurate GPS-height measurements: (a) LSRF point cloud with GPS-heights. (b) LSRF point cloud with barometric heights. 


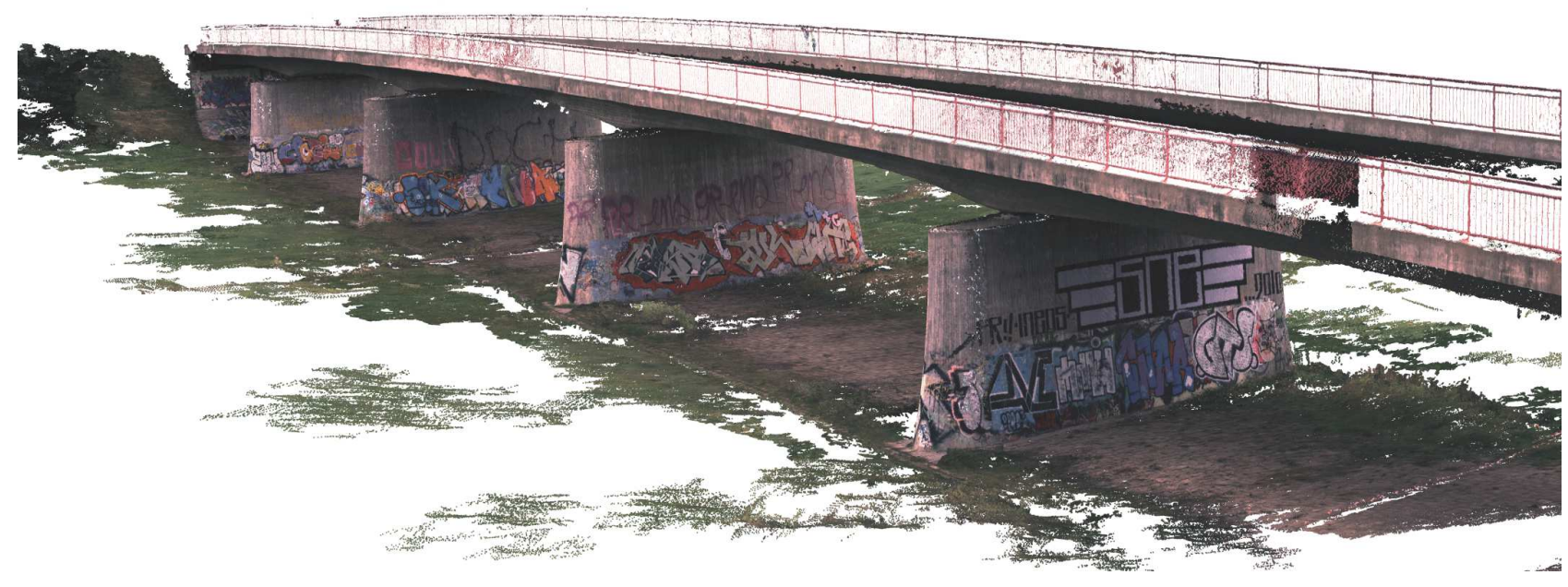

Figure 8: RGB-colored 3D point cloud from SfM.

\section{3D POINT CLOUDS BASED ON IMAGE DATA}

\subsection{Acquisition and data processing}

In a second flight campaign, the bridge mentioned above was imaged by a RGB camera rigidly mounted on the UAV. The entire flight-time for two flights alongside the bridge plus flights around all four bridge pillars was $80 \mathrm{~min}$. About 500 images were captured with an overlap of at least $80 \%$ in flight direction. To ensure an optimal image quality including sharpness and homogenous brightness during the complete flight, the balance between aperture, exposure time and gain is important. A too long exposure time leads to blur effects caused by the motion and vibration of the flying platform. To receive optimal pictures for further processing with SfM methods, aperture and gain was coordinated. During this procedure, too small aperture values as well as too high gain values were avoided. The bridge was reconstructed on basis of images only by SfM-tools, in this case with the commercial software package Agisoft Photoscan. The general principle of SfM has often been presented in literature like (Koenderink and Van Doorn, 1991), (Beardsley et al., 1997) and (Pollefeys et al., 2000). For the presented reconstruction no additional prior informations such as GPS positions or image sequence details are used during the matching process. SfM techniques provide 3D object informations from overlapping image sequences. Knowlegde about inertial information from the captured object or camera poses are not necessary but can reduce processing time and wrong camera pose estimation. Initially, a feature point measurement in every image was carried out by a feature detection operator such as SIFT (Lowe, 1999) or SURF (Bay et al., 2008). After pairwise matching based on detected features, the exterior orientations of the images and a sparse point cloud were reconstructed. The final results of the procedure were a dense point cloud and refined exterior orientations.

\subsection{Result}

The resulting RGB-colored 3D point cloud shown in Figure 8 consists of 13 million points (bridge only) and represents the bridge in a high degree of detail. To analyze the geometrical accuracy, a comparison with the TLS reference point cloud was performed. Therefore the same procedure as in section 4.2 was carried out. The color-coded deviations between both point clouds are shown in Figure 9a. The deviations RMS value is $4.9 \mathrm{~cm}$, with $2 / 3$ of the $S f M$ point cloud having deviations less than $4.1 \mathrm{~cm}$ (Figure 9b). These deviations can be explained on the one hand by a suboptimal capture setup (only one row of images), which has a bad effect to the SfM error propagation. And on the other hand the largest deviations can be found at points near discontinues underneath the bridge (Figure 9c) caused by a lack of texture and brightness in the images. The shown result is a good data basis for any further processing to a meshed 3D visualization for use within $3 \mathrm{D}$ city model. The quality of the point cloud is also sufficient for measurements in the object and for building reconstruction. The processing and evaluation was performed with the open source project CloudCompare. Beyond measuring in the point cloud, there is the possibility to use the images (including their improved georeferencing information) for visual inspection such as damage detection/localization on buildings (Figure 9d).

\section{CONCLUSION}

The paper presents and compare the generation of 3D point clouds on the basis of data from a low-cost LSRF and a RGB camera mounted on a UAV. Properties of the resulting point clouds are presented in Table 1. A processing chain from raw data to 3D object points was shown in an overview as well as remarks for improvements was shown. A comparison of the LSRF with a TLS reference point cloud provides a good estimate of the accuracy potential. A real-time generation of a coarse point cloud based on measurement data of the presented LSRF system is possible. The resulting point cloud with RMS deviations of about $32 \mathrm{~cm}$ is sufficiently to give an overview of the measured object and may also be used for path planning and obstacle avoidance. However, the quality of the LSRF point cloud depends directly on the quality of the UAV's positioning devices, so that the navigation solution should be improved for better results. In a second campaign, the bridge was captured by a RGB camera mounted on a UAV. On basis of the overlapping images, a RGB-colored $3 \mathrm{D}$ point cloud was generated. A comparison with the TLS reference point cloud showed a high geometrical quality in the order of $5 \mathrm{~cm}$.

\begin{tabular}{|l|c|c|}
\hline Sensor & $\begin{array}{c}\text { Hokuyo } \\
\text { UTM-30LX-EW }\end{array}$ & $\begin{array}{c}\text { Prosilica } \\
\text { GT3300C }\end{array}$ \\
\hline point attributes & intensity & RGB color \\
processing time & real time capable & several hours \\
RMS point accuracy & $0.32 \mathrm{~m}$ & $0.049 \mathrm{~m}$ \\
total points (bridge only) & $1 \mathrm{mil}$ & $13 \mathrm{mil}$ \\
\hline
\end{tabular}

Table 1: Properties of LSRF and SfM point clouds. 

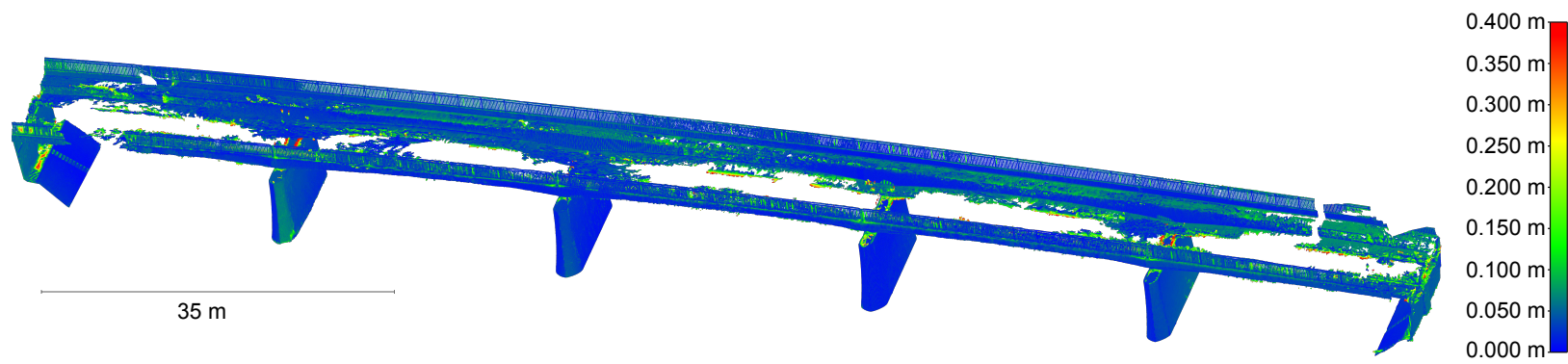

(a)

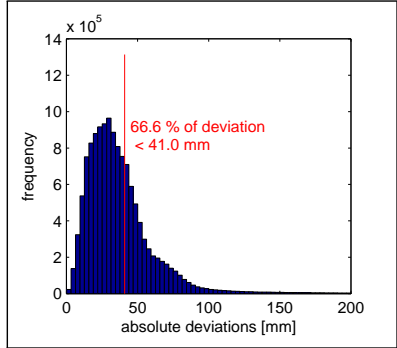

(b)

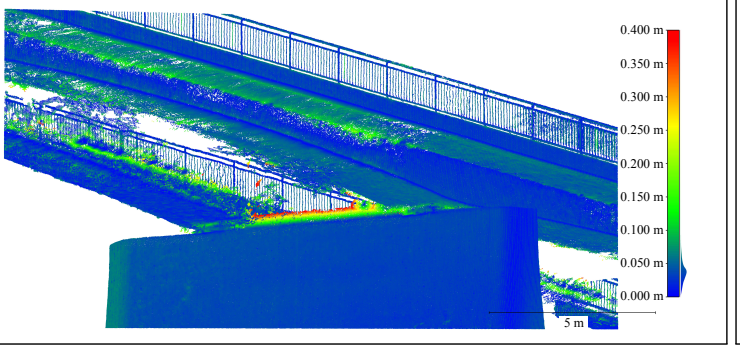

(c)

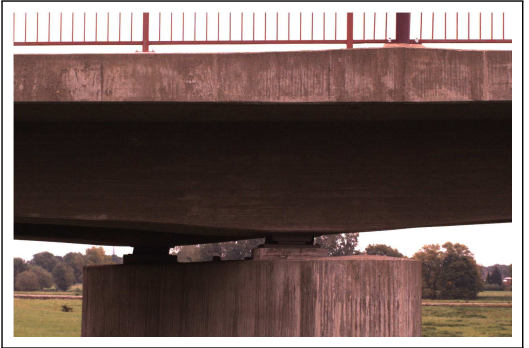

(d)

Figure 9: (a) Color-coded absolute distances between SfM and TLS point clouds obtained by CloudCompare. (b) Histogram of absolute distances. (c) Section of the SfM point cloud showing good points (blue), inaccurate points (red) and holes in the point cloud. (d) Images of the SfM network can be used for visual inspection for instance to detect and localize damage on buildings.

\section{OUTLOOK AND FUTURE WORK}

Further work will be focus on the enhancement of both point clouds. In case of the SfM point cloud, the acquisition of the poorly illuminated bridge areas will be improved to close the gaps in the resulting point cloud. Further step will be coarse georeferencing by using UAV-positioning data. For the LSRF point cloud, improvements in the navigation solution might be achieved by using a navigation camera and a visual SLAM algorithm. Thus the resulting point cloud will be more accurate, and scanning underneath the bridge will be possible due to the reduced vulnerability to GPS signal loss. In addition to that, a reasonable calibration strategy for the IMU will be worked out. An other aspect of future work will be a LSRF strip adjustment for optimization of the point cloud geometry. Furthermore, an integrated point cloud generated by a quality parameter ruled merging process of the SfM and LSRF point clouds will improve the completeness and consistency of the results.

\section{ACKNOWLEDGEMENTS}

The research work presented in this paper has been funded by the European Social Fund (ESF) via Sächsische Aufbaubank (SAB). We would also like to thank our partners in the ADFEX project for their support and the great collaboration.

\section{REFERENCES}

Ahmed, A., Nagai, M., Tianen, C. and Shibasaki, R., 2008. UAV based monitoring system and object detection technique development for a disaster area. International Archives of Photogrammetry, Remote Sensing and Spatial information Sciences 37, pp. 373-377.

Bäumker, M. and Heimes, F., 2001. New calibration and computing method for direct georeferencing of image and scanner data using the position and angular data of an hybrid inertial navigation system. In: OEEPE Workshop, Integrated Sensor Orientation.
Bay, H., Ess, A., Tuytelaars, T. and Van Gool, L., 2008. Speededup robust features (SURF). Computer vision and image understanding 110(3), pp. 346-359.

Beardsley, P. A., Zisserman, A. and Murray, D. W., 1997. Sequential updating of projective and affine structure from motion. International journal of computer vision 23(3), pp. 235-259.

Daftry, S., Hoppe, C. and Bischof, H., 2015. Building with drones: Accurate 3D facade reconstruction using MAVS. arXiv preprint arXiv:1502.07019.

Dorveaux, E., Vissiere, D., Martin, A.-P. and Petit, N., 2009. Iterative calibration method for inertial and magnetic sensors. In: Decision and Control, 2009 held jointly with the 2009 28th Chinese Control Conference. CDC/CCC 2009. Proceedings of the 48th IEEE Conference on, IEEE, pp. 8296-8303.

Eltner, A., Mulsow, C. and Maas, H.-G., 2013. Quantitative measurement of soil erosion from TLS and UAV data. ISPRSInternational Archives of the Photogrammetry, Remote Sensing and Spatial Information Sciences 1(2), pp. 119-124.

Gebre-Egziabher, D., 2007. Magnetometer autocalibration leveraging measurement locus constraints. Journal of aircraft 44(4), pp. 1361-1368.

Georgiev, A. and Allen, P. K., 2004. Localization methods for a mobile robot in urban environments. Robotics, IEEE Transactions on 20(5), pp. 851-864.

Heredia, G., Caballero, F., Maza, I., Merino, L., Viguria, A. and Ollero, A., 2009. Multi-unmanned aerial vehicle (UAV) cooperative fault detection employing differential global positioning (DGPS), inertial and vision sensors. Sensors 9(9), pp. 75667579.

Hokuyo, 2014a. Product Information UTM-30LX-EW. Hokuyo Autmatic Co., Ltd., Japan.

Hokuyo, 2014b. UTM LaserSpot Information. Hokuyo Autmatic Co., Ltd., Japan. 
Israel, M., 2011. A UAV-based roe deer fawn detection system. International Archives of the Photogrammetry, Remote Sensing and Spatial Information Sciences 38(1/C22), pp. 51-55.

Koenderink, J. J. and Van Doorn, A. J., 1991. Affine structure from motion. Journal of the Optical Society of America A 8(2), pp. 377-385.

Lowe, D. G., 1999. Object recognition from local scale-invariant features. In: Computer vision, 1999. The proceedings of the seventh IEEE international conference on, Vol. 2, Ieee, pp. 11501157.

Mader, D., Westfeld, P. and Maas, H.-G., 2014. An integrated flexible self-calibration approach for 2D laser scanning range finders applied to the Hokuyo UTM-30LX-EW. ISPRSInternational Archives of the Photogrammetry, Remote Sensing and Spatial Information Sciences 1, pp. 385-393.

Metni, N., Pflimlin, J.-M., Hamel, T. and Souères, P., 2006. Attitude and gyro bias estimation for a VTOL UAV. Control Engineering Practice 14(12), pp. 1511-1520.

Pollefeys, M., Koch, R., Vergauwen, M., Deknuydt, A. A. and Van Gool, L. J., 2000. Three-dimensional scene reconstruction from images. In: Electronic Imaging, International Society for Optics and Photonics, pp. 215-226.

Roca, D., Armesto, J., Lagüela, S. and Díaz-Vilariño, L., 2014. Lidar-equipped UAV for building information modelling. ISPRSInternational Archives of the Photogrammetry, Remote Sensing and Spatial Information Sciences 1, pp. 523-527.

Stempfhuber, W. and Buchholz, M., 2011. A precise, low-cost RTK GNSS system for UAV applications. International Archives of Photogrammetry, Remote Sensing and Spatial Information Science 38, pp. 1-C22. 